\title{
Morphology Effects of Cap-shaped Silver Nanoparticle Films as a SERS Platform
}

\author{
Hiroyuki TAKEI ${ }^{* * * \dagger}$ and Takayuki ОКАMото $* * *$ \\ *Faculty of Life Sciences, Toyo University, Itakura, Gunma 374-0193, Japan \\ **Bio-Nanoelectronics Centre, Toyo University, Kawagoe, Saitama 350-8585, Japan \\ ***RIKEN, 2-1 Hirosawa, Wako, Saitama 351-0198, Japan
}

\begin{abstract}
In this paper, we evaluate randomly adsorbed cap-shaped silver nanoparticles for applications to surface-enhanced Raman spectroscopy, SERS. They were prepared by depositing silver on top of surface-adsorbed monodisperse $\mathrm{SiO}_{2}$ nanospheres, in a manner similar to the method for preparing metal film on nanosphere, MFON, but one major difference lies in the fact that nanospheres are randomly adsorbed rather than as a close-packed MFON. With random MFON, it is possible to incorporate nanospheres with more than one size. Mixing has been found to increase SERS performance. More specifically, by using 50 and $100 \mathrm{~nm}$ nanospheres, we found that substrates containing both types outperform substrates prepared from $100 \%$ of either 50 or $100 \mathrm{~nm}$ nanospheres. As evaluated by spectrophotometry, this increase could not be attributed to an increase in the extinction coefficient of the substrate at the irradiation wavelength of SERS measurements.
\end{abstract}

Keywords Surface-enhanced Raman spectroscopy, metal film on nanospheres, random structure, heterogeneity

(Received November 20, 2015; Accepted January 8, 2016; Published March 10, 2016)

\section{Introduction}

SERS is one of the oldest optical analytical techniques based on localized surface-plasmon resonance (LSPR) effects associated with noble metal nanostructures/particles. Since its initial discovery in the $1970 \mathrm{~s},{ }^{1,2}$ the importance of metal nanostructure/ particle structure has become recognized also for infrared spectroscopy and fluorescence spectroscopy. ${ }^{3-5}$ Among these techniques, infrared spectroscopy has benefitted from enhancement ratios on the order of one hundred, and surfaceenhanced fluorescence spectroscopy, also known as metalenhanced fluorescence, can claim similar enhancement ratios. Meanwhile, enhancement ratios upward of $10^{10}$ have been reported by a large number of groups for SERS, ${ }^{6}$ enabling detection of a single molecule. While SERS is about to make an impact in the field of analytical chemistry, ${ }^{7}$ a number of technical issues need to be addressed before SERS becomes a routine technique. These are principally reproducibility, stability in the sense of a long shelf-time, and preparation costs.

There are a number of excellent reviews covering various approaches toward requisite substrate preparation ${ }^{8-10}$ so that it suffices to mention that from the initial substrate prepared by electrochemical etching of a silver electrode, advanced nanofabrication tools such as electron beam lithography have been summoned to the task. When more than one particle is located within a tight confine or placed on a metallic surface, the region between them plays a significant role. These "hot spots" are thought to be overwhelmingly responsible for signal enhancement. As a matter of fact, in a system consisting of

$\dagger$ To whom correspondence should be addressed.

E-mail: h_takei@toyo.jp isolated metal particles, dimers, and other higher-order clusters, isolated particles seem to contribute very little to the overall signals. ${ }^{11-15}$

Through experiments and simulations, it is becoming clear what would constitute an effective "hot spot". The gap must be less than $10 \mathrm{~nm}$ wide and preferably formed by two elongated silver features. So-called metal film on nanosphere, MFON, is one of the promising candidates to prepare such features. Van Duyne and his co-workers are some of the earliest to implement this scheme as an extension of nanosphere lithography, NSL. In NSL, a close-packed array of monodisperse nanospheres is first formed on a substrate. After a metal is deposited, the nanospheres are removed, leaving behind an array of quasitriangular features. ${ }^{16}$ With MFON, the substrate is used without removing the metal-coated nanospheres. It is more suitable for formation of narrower gaps. When the angle of deposition is perpendicular to the substrate surface, the gap between adjacent particles tend to fill up with the metal, but with angled deposition, the sphere on one side serves as a shadow to create a gap in the metal film. ${ }^{17}$ MFON has been demonstrated to be an effective SERS substrate. ${ }^{18-22}$

In principle, MFON is prepared with close-packed nanospheres, but metal particles can also be formed atop a monolayer of randomly adsorbed nanospheres. ${ }^{23}$ Some of the features associated with random MFON are (1) spheres do not have to be highly monodisperse, (2) it is possible to mix nanospheres with different diameters to modulate extinction spectra, (3) nanosphere adsorption density can be controlled to modulate the interaction among particles, and (4) a random nanosphere monolayer can be formed over surfaces other than a perfectly flat surface, important in industrial applications. We have explored potentials of random MFON in connection with localized surface plasmon resonance (LSPR) sensing, ${ }^{23-27}$ 
surface-enhanced fluorescence detection, ${ }^{28,29}$ enhanced colorimetric detection, ${ }^{30}$ and surface-enhanced Raman spectroscopy. ${ }^{31}$ Figure S1 (Supporting Information) shows a schematic side view of such a structure (A) and an SEM image of a monolayer of densely adsorbed nanospheres $100 \mathrm{~nm}$ in diameter (B). As with close-packed MFON, random MFON allows metal nanoparticles to be formed from any metal that can be vacuum-deposited. We have prepared $\mathrm{Au}, \mathrm{Ag}$ and $\mathrm{Cu}$ with apparent optical density exceeding two, with resonant peaks having FWHM of approximately $100 \mathrm{~nm}$ in the reflection mode. ${ }^{23,24}$ The peak wavelength can be tuned over the entire visible region as well as into the near infrared region by selection of the nanosphere diameter in the range from tens of $\mathrm{nm}$ to hundreds of $\mathrm{nm}$ and metal deposition thickness from $5 \mathrm{~nm}$ to tens of nm. Baia et al. has also shown such dependence on the deposition thickness with close-packed MFON. ${ }^{21,32}$ The gap distance among adjacent particles also plays an important role. ${ }^{33}$ Gap control can be achieved by dry-etching the surface-adsorbed sphere before deposition of the metal on top. ${ }^{24,34}$

In this paper, first we show Raman signals from the random $\mathrm{AgFON}, \mathrm{Ag}$ film on nanosphere are significantly greater than those from a number of control surfaces such as a monolayer of bare $\mathrm{SiO}_{2}$ nanospheres on a glass slide, a glass slide and a glass slide covered by $\mathrm{Ag}$, using rhodamine $6 \mathrm{G}, \mathrm{R} 6 \mathrm{G}$, as a model compound. Then we will show how changing the Ag deposition thickness and the nanosphere diameter affect both the extinction spectrum and SERS spectrum. Then, we demonstrate unique features of random AgFON by modifying the sphere adsorption density. It can be controlled by the concentration of $\mathrm{MgCl}_{2}$ that was added to the sphere suspension during the adsorption process. Salt induced partial aggregation of spheres, thus increasing the adsorption density. Substrates so prepared have been characterized by optical extinction, scanning electron microscopy and SERS measurements. Another approach was to mix nanospheres of different diameters. Specifically, we mixed nanospheres with 50 and $100 \mathrm{~nm}$ diameters. Resulting mixture substrates show extinction spectra that were drastically modified. SERS spectra were correspondingly different. Such results are in accord with the knowledge that interactions among particles are critical for effective surface enhancement, and in recent years, multi-scale systems composed of particles of varying sizes have been reported as particularly effective. ${ }^{35,36}$ The random MFON system consisting of nanospheres of different diameters would be a good way to demonstrate and exploit the efficacy of multi-scale systems.

\section{Experimental}

\section{Reagents and chemicals}

$\mathrm{SiO}_{2}$ nanospheres with nominal diameters of 50, 100, and $150 \mathrm{~nm}$ (Polysciences Inc., Silica Microspheres, Cat. No. 24040, 24041, and 24320, respectively) were used without further processing. Unless specified otherwise, $100 \mathrm{~nm}$ nanospheres were used. Glass slides were purchased from Matsunami (Osaka, Japan, FF-003) which were treated with 3-aminopropyltrimethoxysilane, APTMS (Wako, Osaka, Japan, Cat. No. 323-74352). A silicone sheet with a regular array of holes with $3 \mathrm{~mm}$ diameter (Invitrogen, CultureWell ${ }^{\mathrm{TM}}$ Multiwell Chambered Coverslip, Cat. No. C-24780) was used to confine sphere suspension on a glass slide. Magnesium chloride $\left(\mathrm{MgCl}_{2}\right)$ was purchased from Wako (Cat. No. 139-00185). Silver pellets for vacuum evaporation were purchased from Nilaco Corporation (99.999\% purity, Cat. No. 400025). Rhodamine 6G (R6G) was purchased from Sigma-Aldrich (Cat. No. R4127-5G).

\section{Preparation of random AgFON}

A glass slide glass was exposed to an aqueous APTMS solution ( $1 \mathrm{vol} \%$ ) for $2 \mathrm{~min}$ and then blow-dried. Then, a silicone sheet was placed on the glass slide to form wells into which nanosphere suspension was added. Nanospheres began to adsorb onto the glass slide surface immediately with the full coverage achieved in tens of seconds. The extent of the final coverage can be modulated by addition of $\mathrm{MgCl}_{2}$. An increase in the ionic strength leads to screening of the electrostatic repulsive force among nanospheres, leading to an increased adsorption density. Appropriate concentrations ranged from a few $\mathrm{mM}$ to a few hundred mM. When AgFON with multiple nanospheres was needed, a nanosphere suspension mixture was prepared by mixing various nanosphere suspensions in required volume ratios before being added to a glass slide. The numbers of $\mathrm{Ag}$ nanoparticles per unit area were recorded from SEM images taken with the JSM-7400 F (JEOL, Tokyo, Japan). Three regions with an equal area were arbitrarily chosen from each image, and the average number of nanospheres counted in three regions was used to obtain the adsorption density.

After surface-adsorbed nanospheres were dried, they were placed into a vacuum evaporator, the VFR-200M/ERH (ULVAC KIKO, Inc., Miyazaki, Japan). Ag evaporation was carried out with the vacuum pressure of $5 \times 10^{-3} \mathrm{~Pa}$ or better at a typical evaporation rate of $0.2 \mathrm{~nm} / \mathrm{s}$.

\section{SERS measurements}

To assure that relative merits of various substrates were actually evaluated, substrates to be compared were prepared on the same day and evaluated at the same time, within a week after preparation. Rhodamine $6 \mathrm{G}$ at the concentration of $10 \mathrm{mM}$ in ethanol, was used as a model compound. Substrates were immersed in the R6G solution for 5 min. After substrates were removed from the R6G solution, excess R6G solution was immediately removed by blow-drying to prevent uneven accumulation of R6G molecules on the surface. The R6G concentration was intentionally set high to assure as much uniformity and reproducibility as possible even though it was possible to detect R6G with concentrations well below $1 \mu \mathrm{M}$. All Raman spectra were obtained with the Horiba Jovin Yvon HR 800 Raman microscope (Horiba, Kyoto, Japan). Spectra were obtained with $514.5 \mathrm{~nm}$ irradiation at the intensity of $50 \mu \mathrm{W}$, with a $10 \times$ objective in place. The exposure time was $5 \mathrm{~s}$, and three consecutive spectra were averaged. This condition was chosen to minimize the effect of bleaching. Increasing the irradiation intensity by another order of magnitude or use of a $100 \times$ microscope objective was found to lead to a noticeable decay in the signal intensity upon irradiation. In most figures to follow, when more than one Raman spectrum are shown, the base lines have been shifted in the vertical direction to facilitate viewing of the multiple spectra.

Extinction spectra were obtained with the USB 4000 Fiber Optic Spectrometer from Ocean Optics in reflection mode. A custom bifurcation fiber was used in combination with the LS-1 Tungsten Halogen Light Source (Ocean Optics) for irradiation. The single end of the fiber bundle consisted of a 1-mm fiber for irradiation surrounded by other 1-mm fibers for guiding the reflected light to the spectrometer. For a reference surface, a plane $\mathrm{Ag}$ film with an equivalent thickness was used. For example, if nanospheres were covered with $20 \mathrm{~nm}$ of $\mathrm{Ag}$, then a $20-\mathrm{nm}$ thick Ag film, on a glass slide was used as a reference. 


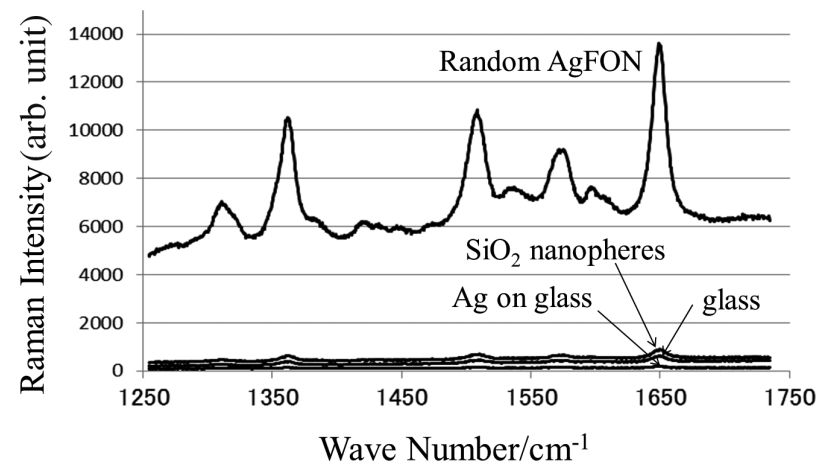

Fig. 1 Raman spectra of rhodamine 6G from various substrates. Signals were obtained from random AgFON, a monolayer of bare nanospheres (nanospheres), a glass slide (glass) and a glass slide covered by $160 \mathrm{~nm}$ thick Ag (Ag on glass).

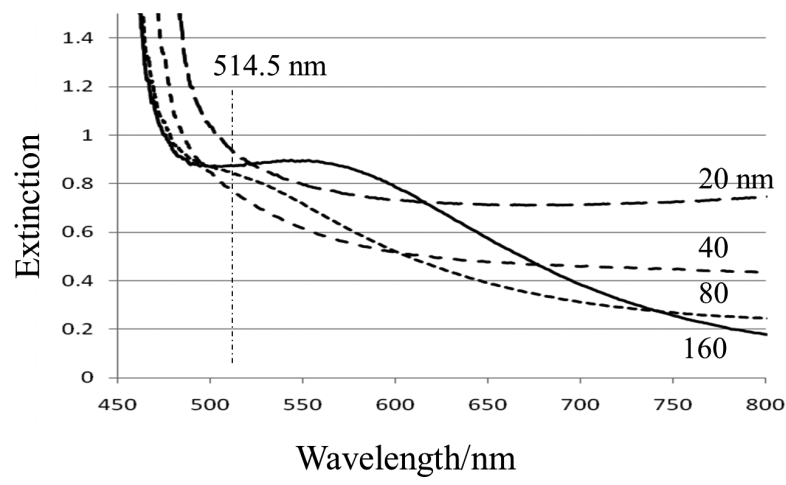

Fig. 2 Dependence of the extinction spectrum on the Ag deposition thickness.

\section{Results and Discussion}

The SEM image in Fig. S1 (Supporting Information) (B) represents a typical random $\mathrm{AgFON}$ substrate. Here $80 \mathrm{~nm}$ of $\mathrm{Ag}$ was evaporated on a monolayer of densely adsorbed $100 \mathrm{~nm}$ diameter nanospheres. With the $\mathrm{Ag}$ deposition thickness of $80 \mathrm{~nm}$, almost all nanospheres were in direct contact with their neighbors. These were electrically isolated from the underlying substrate; when a TEM micrograph was obtained of nanoparticles dislodged from the glass slide, one side was free of metal. ${ }^{24}$ Here we make a distinction between a nanosphere and a nanoparticle as follows. The former refers to a bare $\mathrm{SiO}_{2}$ nanosphere whereas the latter refers to a nanosphere coated with Ag. AgFON refers to a dense monolayer of Ag nanoparticles.

Figure S2 (Supporting Information) is an image of a glass slide with $\mathrm{Ag}$ nanoparticles within the central hexagonal region (360 $\mu \mathrm{m}$ across) and bare nanospheres in the surrounding area, under white light illumination from top. It is clear that $\mathrm{Ag}$ nanoparticles are significantly superior scatterers than bare nanospheres.

Figure 1 shows R6G Raman spectra from various substrates; $100 \mathrm{~nm}$ AgFON with $160 \mathrm{~nm}$ thick Ag, bare nanospheres without Ag, a glass slide, and a glass slide covered by $160 \mathrm{~nm}$ of Ag.

The spectrum from AgFON was by far the most prominent. For this set of measurements, all four substrates were prepared on a single glass slide, side by side, so that they were all

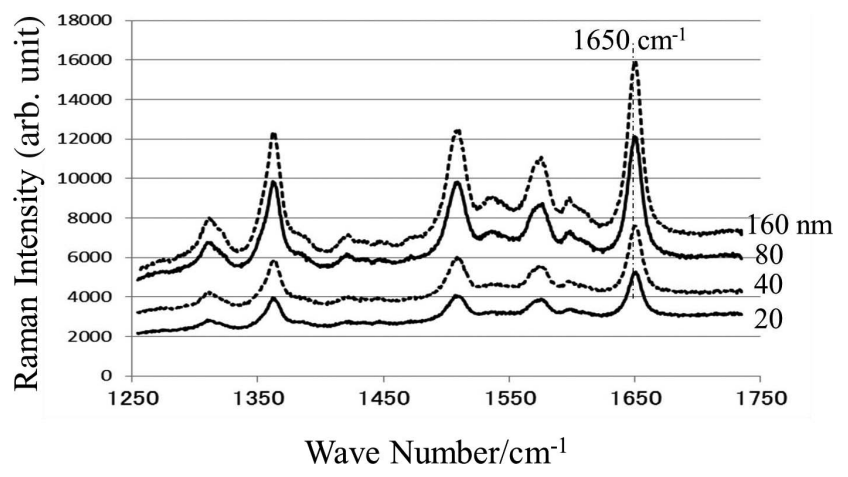

Fig. 3 Dependence of the R6G SERS spectrum on the Ag deposition thickness.

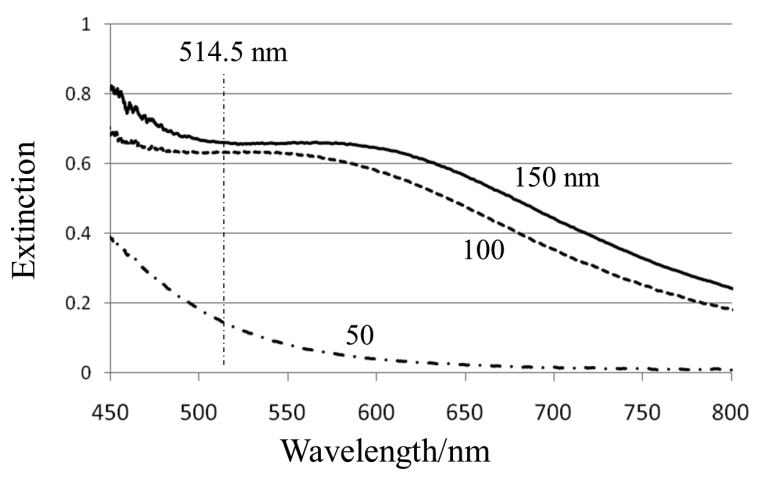

Fig. 4 Dependence of the extinction spectrum on the nanosphere diameter. The deposition thickness is $160 \mathrm{~nm}$.

immersed in R6G for the same duration at the same time, assuring quantitative comparison.

Next we show the effect of modifying the $\mathrm{Ag}$ deposition thickness. Figure 2 shows extinction spectra of $100 \mathrm{~nm}$ AgFON with 20, 40, 80, and $160 \mathrm{~nm}$ thick Ag. The broken line indicates the irradiation wavelength of $514.5 \mathrm{~nm}$. At this wavelength, extinction varied only from the low of 0.76 and high of 0.92 , and there was hardly any peak that might be expected to result in a resonant effect.

Then, we show R6G SERS spectra from the same set of substrates in Fig. 3. With the increasing deposition thickness, there was a clear increase in the Raman intensity. Judging from intensities of the $1650 \mathrm{~cm}^{-1}$ peak, the $160 \mathrm{~nm}$ deposition was found to lead to a further increase by 3.6 fold. It would be expected that $100 \mathrm{~nm}$ nanospheres would be buried completely under $160 \mathrm{~nm}$ thick silver, but as SEM images in Fig. S3 (Supporting Information), showing nanospheres after deposition of 100 and $500 \mathrm{~nm} \mathrm{Ag}$ for $\mathrm{A}$ and $\mathrm{B}$, respectively, reveal, even with $500 \mathrm{~nm}$ of $\mathrm{Ag}$ deposited, nanospheres retain their original morphology.

Another parameter that has a large influence was the sphere diameter. Extinction spectra of AgFON prepared from 50, 100, and $150 \mathrm{~nm}$ nanospheres with $160 \mathrm{~nm}$ thick $\mathrm{Ag}$ are shown in Fig. 4. Corresponding SERS spectra are shown in Fig. 5. Here, 100 and $150 \mathrm{~nm}$ nanospheres show prominent SERS spectra, which also exhibited correspondingly more prominent extinctions.

Next we show results obtained by altering the nanosphere adsorption density. As mentioned in the Experimental section, the nanosphere adsorption density could be controlled by 


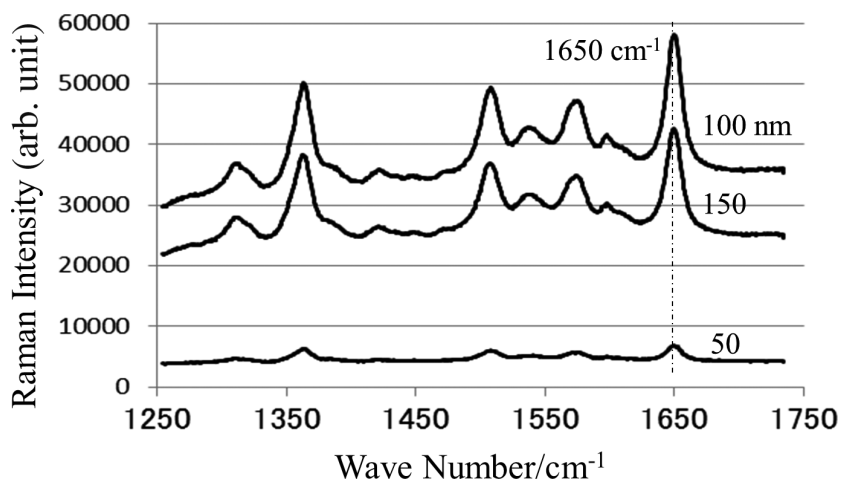

Fig. 5 Dependence of the R6G SERS spectrum on the nanosphere diameter. The deposition thickness is $160 \mathrm{~nm}$.

changing the $\mathrm{MgCl}_{2}$ concentration. As the adsorption process was quite rapid, with the saturation coverage achieved well under a few minutes, signals from AgFON prepared with the incubation time of $10 \mathrm{~min}$ were quite reproducible. On the other hand, other parameters such as temperature, surface pre-cleaning, lot-to-lot variation of the sphere suspension all had insignificant roles in the final adsorption density. Figure S4 (Supporting Information) show SEM images of AgFON prepared with various $\mathrm{MgCl}_{2}$ concentrations. Images $\mathrm{A}, \mathrm{B}, \mathrm{C}$, $\mathrm{D}$, and $\mathrm{E}$ correspond to substrates prepared with $0,3,15,62$, and $250 \mathrm{mM}$ of $\mathrm{MgCl}_{2}$. With the increasing concentration, the adsorption density increased noticeably from 0 to $15 \mathrm{mM}$, while a small number of spheres began to adsorb on top of the first layer for the concentration around $15 \mathrm{mM}$. Their extinction spectra are shown in Fig. S5 (Supporting Information). Most interestingly, the sparsest substrate with no $\mathrm{MgCl}_{2}$ added resulted in the most pronounced peak. Increasing the $\mathrm{MgCl}_{2}$ concentration was found to lead to a reduction in the overall extinction. Obviously as the density increased, collectively nanoparticles behave more like a continuous film rather than isolated nanoparticles so that the space around nanoparticles played an important role in making nanoparticles more efficient scatterers. R6G SERS spectra from the same set of substrates are shown in Fig. S6 (Supporting Information), with the inset showing intensities of the $1650 \mathrm{~cm}^{-1}$ peak.

As the last type of morphology explored in this paper, we show results from mixture substrates, consisting of nanospheres of different sizes. Mixture substrates were made by first mixing 100 and $50 \mathrm{~nm}$ nanosphere suspensions (5.59 and 5.9\% aqueous suspensions, respectively) with various volume ratios. The volume ratio was set at 100:0, 80:20, 60:40, 40:60, 20:80, and $0: 100$. Resulting substrates, henceforth to be referred to as 100 , $80,60,40,20$ and $0 \%$ substrates, exhibited widely varying extinction spectra as shown in Fig. 6 where the Ag thickness was $160 \mathrm{~nm}$. There was a rapid drop in extinction at $514.5 \mathrm{~nm}$ with an increase in the proportion of the smaller nanosphere.

R6G SERS spectra from these substrates are shown in Fig. 7. Both 100 and $80 \%$ substrates were characterized by equally strong scattering, but with a further increase in the proportion of the smaller nanosphere, scattering rapidly diminished. Having realized that the morphology of the 100 and $80 \%$ substrates were quite distinct, we decided to take a closer look at this mixture ratio regime. Figure 8 shows SEM images of 100, 96, $92,88,84$ and $80 \%$ substrates, from $\mathrm{A}$ to $\mathrm{F}$.

In all, the proportion of the smaller nanoparticles to that of the larger nanoparticles significantly surpassed what would be expected from the volume ratio of the original nanosphere

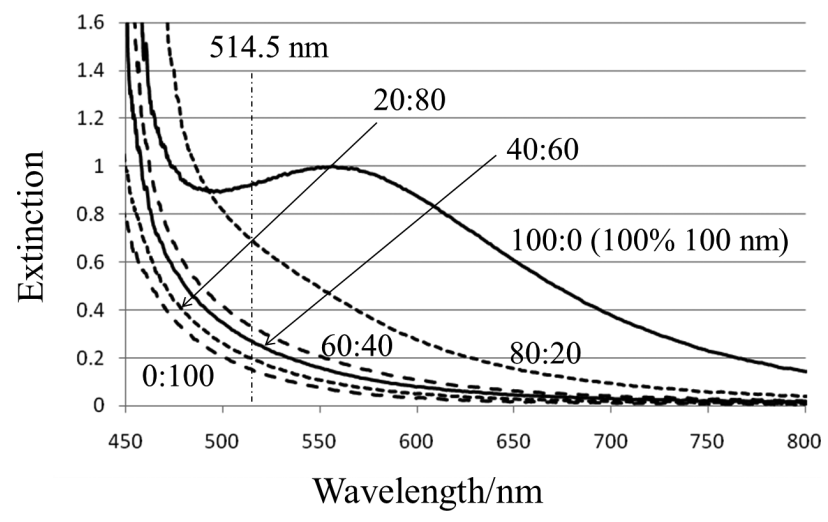

Fig. 6 Extinction spectra of the mixture substrates consisting of 10 and $50 \mathrm{~nm}$ nanospheres. The ratio ranges from $100 \% 100 \mathrm{~nm}$ nanospheres (100:0) to $100 \% 50 \mathrm{~nm}$ nanospheres $(0: 100)$.

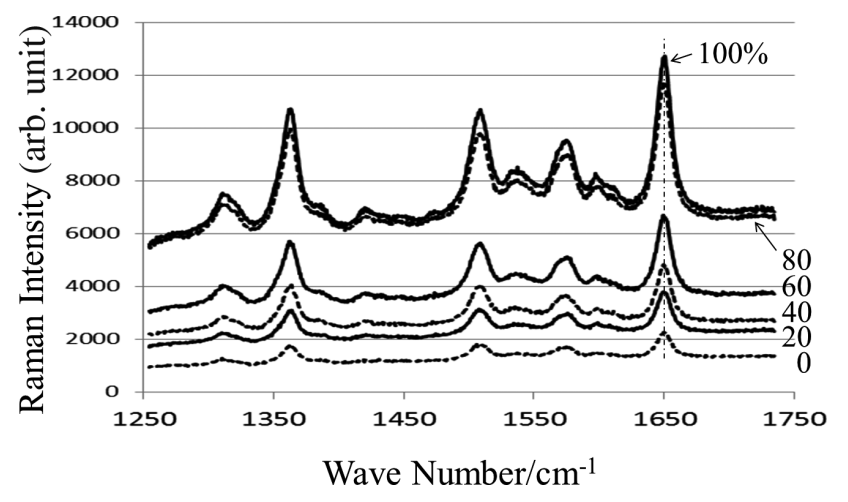

Fig. 7 Dependence of the R6G SERS spectrum on the proportion of $100 \mathrm{~nm}$ nanospheres.

mixture suspension. Apparently, the $50 \mathrm{~nm}$ nanosphere adsorbed onto the glass surface more preferentially. Specifically, the adsorption density of nanospheres for the $100 \%$ substrate was 48.3 per $\mu \mathrm{m}^{2}$ whereas for the $80 \%$ substrate, densities were 17.4 and 60.9 per $\mu \mathrm{m}^{2}$ for the larger and smaller nanospheres, respectively. Data for other substrates are shown as a bar graph in Fig. S7 (Supporting Information). With the mixture ratio of $96 \%$, the density of the smaller nanoparticles was already greater than that of the larger nanoparticles. As the proportion of the $50 \mathrm{~nm}$ nanosphere increased, one would expect a reduction in SERS signal intensities because the smaller nanoparticles was significantly less effective as has been seen in Fig. 5. R6G SERS spectra of these substrates are shown in Fig. 9. Intensities of the $1650 \mathrm{~cm}^{-1}$ peak are shown as a bar graph in the inset.

There have been a number of reports on use of MFON as a substrate for SERS, and its effectiveness has been proven. MFON has advantages of simplicity in preparation, controllability in the particle size and thickness and is superbly suited for formation of gaps. ${ }^{18-22,32}$ Van Duyne et al. were some of the earliest workers to demonstrate its utility by showing SERS spectra of BPE and pyridine. ${ }^{18}$ Later, they explored the diameter dependence by employing nanospheres with diameters ranging from 390 to $600 \mathrm{~nm}$. The enhancement as high as 200000 over control surfaces was reported. ${ }^{19}$ The size dependence was also explored by Baia et al. ${ }^{21,32}$ In these papers, nanospheres were coated by gold. In their case, substrates were prepared with nanospheres 220 or $400 \mathrm{~nm}$ in diameter and evaluated by depositing R6G. While it is generally more 

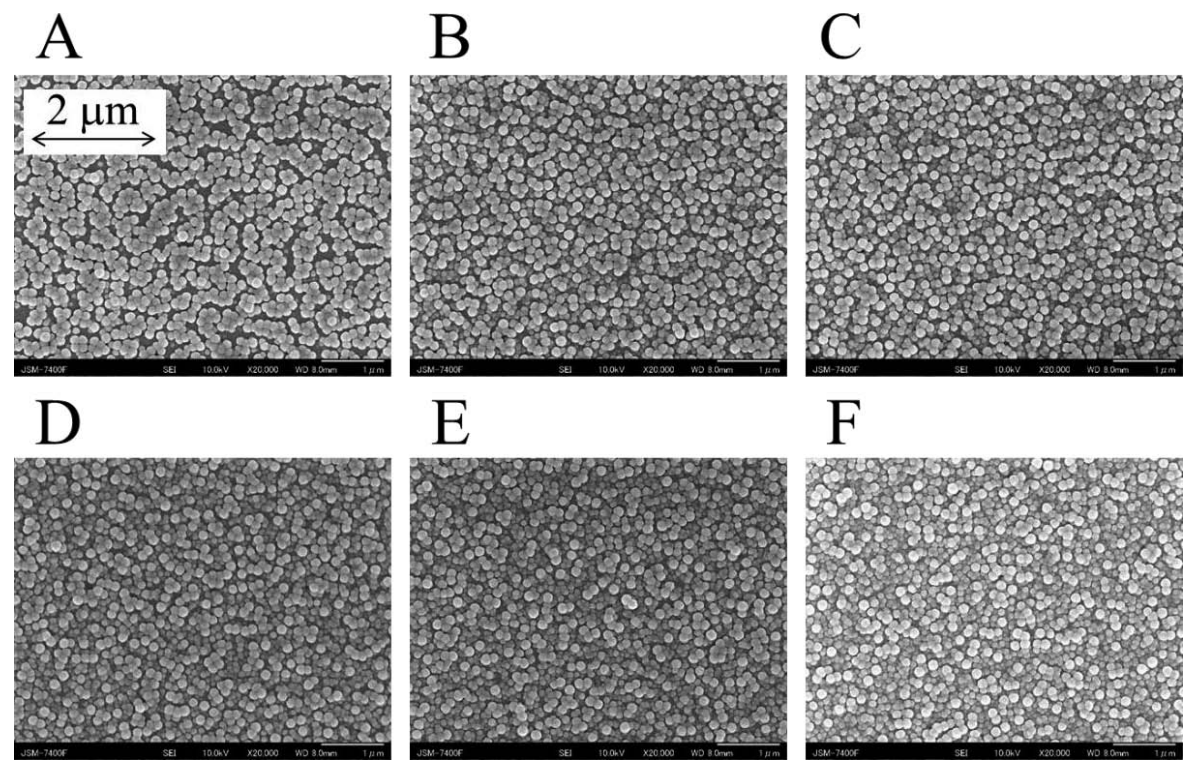

Fig. 8 SEM images showing mixture substrates prepared with various mixed nanosphere suspensions, with A, B, C, D, E, and F corresponding to $100,96,92,88,84$, and $80 \%$ of $100 \mathrm{~nm}$ nanospheres in volume proportion.

difficult to use smaller diameter nanospheres for making a close-packed MFON, Jeong et al. managed to form such an array with nanospheres with $100 \mathrm{~nm}$ diameter. ${ }^{22}$

The fact that metal deposited on surface-adsorbed nanospheres is shaped like a half-shell is considered important. Nano coreshells consisting of a metal nanoparticle with a dielectric core have been explored in detail. In comparison to solid metal particles, they are apparently more effective for SERS.9,37 Improvement in the performance can also result from reduced symmetry.

With close-packed MFON, it is reasonable to assume that every junction formed by every pair of two adjacent metal particles would generate a hot spot of more or less equal strength. However, Dlott et al. have reported that of a million such potential junctions, some were significantly hotter than the rest. ${ }^{38}$ In one example, it was speculated that the hottest 63 hot spots contribute some $24 \%$ of the total signal. This shows that even when two junctions look identical under the scrutiny of SEM observation, they make widely different contributions.

Close-packed MFON comes close to the limit of what can be produced with high precision over a macroscopic area; even with the power of electron beam lithography, controlling the geometry of the junction is difficult if the smallest feature dimension is below $10 \mathrm{~nm}$. It is necessary to come up with a way to prepare a practical structure that may provide more effective hot spots, both in terms of the strength of the near-field and number. One answer might be to incorporate a certain amount of randomness to MFON. Random systems have been receiving increasing attention. Some are called aperiodic while some others are referred to as self-similar. Stockmann et al. have described a chain of metal particles whereby the size changes along the length of the chain. ${ }^{39}$ As a surface plasmon propagates along the chain, the optical energy was assumed to become concentrated and tremendously strong hot spots were generated at successive junctions. The enhancement was thought to be particularly effective when the difference in size spans over a large scale. Experimentally, Reinhard and Kneipp have been directing their efforts toward such systems. ${ }^{36,40}$ Reinhard and his co-workers, using electron beam lithography,

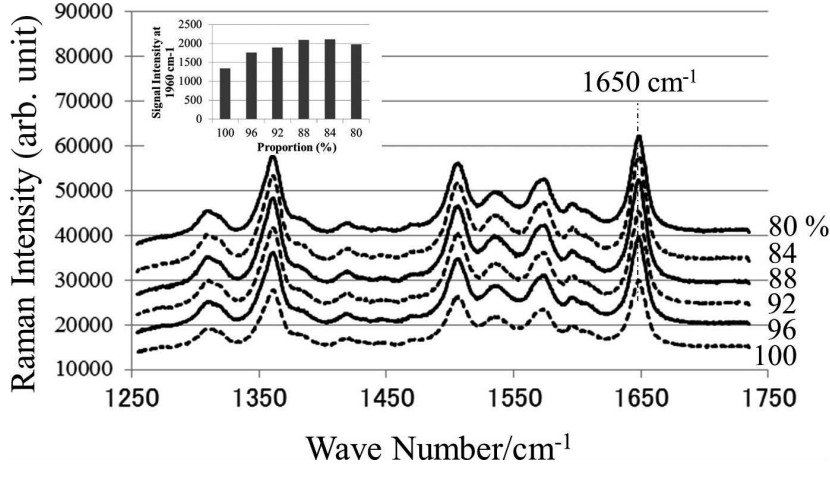

Fig. 9 Dependence of the R6G SERS spectrum on the mixture proportion. The inset shows intensities of the $1650 \mathrm{~cm}^{-1}$ peak.

have created what they call nanocluster arrays, and having "satellite" on larger sphere is particularly effective. ${ }^{36}$ Kneipp et al. have been working with preformed particles which are induced to aggregate by controlling ionic strength. In such systems, even relatively large gaps seem effective. ${ }^{40}$ Baia et al. have made observations in connection with randomness of nanoparticles; in one of their papers, ${ }^{21}$ they made a comparison among SERS signals from three types of domains. Principally, they were carrying out a study on a highly ordered lattice system but when they also measured signals from two other types of domain, random and double layered, they noticed that the random domain gave the highest signals, stating that "the randomly self-assembled nanospheres and nanocavity arrays provide more efficient SERS substrates than regular nanostructures".

Thus, random MFON leads to some interesting results. First of all, our study with modification of the adsorption density shows that the highest density substrate does not lead to the highest total intensity. Of substrates whose SEM images are presented in Fig. S4 (Supporting Information), B with the adsorption density of 50.4 per $\mu \mathrm{m}^{2}$ give the largest $1650 \mathrm{~cm}^{-1}$ 
peak; A and C, with the adsorption density of 43.8 and $55 \mu \mathrm{m}^{-2}$, result in a slightly diminished $1650 \mathrm{~cm}^{-1}$ peak of equal height, within $0.3 \%$ of each other. In terms of the signal intensity per particle, $\mathrm{A}$ is $25 \%$ more effective than $\mathrm{C}$. B follows $\mathrm{A}$ in effectiveness, each particle contributing, however, only as much as $92.7 \%$. As for $\mathrm{D}$ and $\mathrm{E}$, the adsorption density is higher, but the overall SERS intensity diminishes as can be seen in the inset in Fig. S6 (Supporting Information). Thus, A with the least number of particles per unit area is most effective by far. This simple observation does not take into account that it is not individual particles that contribute to SERS signals; rather, it is higher-order clusters consisting of more than a single particle that contribute to SERS. In that sense, A might be characterized as having more clusters than the rest of the substrates. Substrates $\mathrm{C}, \mathrm{D}$ and $\mathrm{E}$ consist of more or less a continuous film. In contrast, A and, to some extent, B show clusters that are island like; particle clusters are arranged like a collection of islands.

On the other hand, thicker Ag deposition should result in continuous films rather than a group of discrete nanoparticles, but as extinction spectra in Fig. 2 show, a peak grew rather than becoming diminished even though the peak did not coincide with the wavelength of the irradiation laser. SERS spectra in Fig. 3 indicate that the SERS intensity also increased with the deposition thickness. While an increase in the total surface area, thus of adsorbed R6G molecules, cannot be excluded, comparison of two images in Fig. S3 (Supporting Information) dictates against that.

Such dependence on the adsorption density has been observed in connection with surface-enhanced fluorescence. ${ }^{29}$ As the density was reduced from the saturation level, the overall signal intensity increased. Especially in terms of the signal intensity per number of particles, this trend was even more noticeable.

When one carries out this simplified analysis to the mixture system, we come up with the following result. If the comparison among nanospheres of different diameters, in terms of the intensity of the $1650 \mathrm{~cm}^{-1}$ peak, shown in Fig. S5 (Supporting Information) is used as the guide, the $50 \mathrm{~nm}$ nanosphere substrate produced only as little as $10.8 \%$ as from the $100 \mathrm{~nm}$ nanosphere substrate even though the nanosphere adsorption density was higher for the $50 \mathrm{~nm}$ nanosphere at $92.5 \mathrm{vs}$. $48.3 \mu \mathrm{m}^{-2}$ for the $100 \mathrm{~nm}$ nanosphere. By taking this into account, a single 50-nm nanoparticle was estimated to contribute only as little as $5.6 \%$ of what a single 100-nm nanoparticle produces. If one combines this piece of information with data in Fig. S7 (Supporting Information), it is possible to obtain a rough estimate for the intensity of the $1650 \mathrm{~cm}^{-1}$ peak for mixture substrates. Figure S8 (Supporting Information) summarizes the result. The light bars reflect actual data while the dark-colored bars show predicted values. Discrepancy between these two groups is quite remarkable; for example, for the $80 \%$ mixture substrate, the actual peak height is 3.4 times the predicted value. Seen from another perspective, the intensity of the $1650 \mathrm{~cm}^{-1}$ peak for the $80 \%$ mixture substrate was $47 \%$ higher than that of the $100 \%$ (fully $100 \mathrm{~nm}$ nanospheres) substrate even though its extinction at the excitation wavelength of $514.5 \mathrm{~nm}$ is $24.9 \%$ less.

Obviously, one cannot treat these substrates as discrete nanoparticles. As is reportedly widely, clustering significantly leads to an increase in SERS spectra. The observation immediately above shows that clustering of nanoparticles of different dimensions are better still. At this stage, we can only speculate what model would predict such behaviors. In the past, we showed that a model based on dimers playing a dominant role could describe extinction spectra of cap-shaped gold nanoparticles in the visible and near-IR regions; ${ }^{27}$ currently we are trying to find out whether more complicated clusters such as trimer or tetramer are more appropriate. At the same time, further work on characterizing gap junction areas within a cluster is needed. Particularly when two nanospheres of significantly different diameters touch each other, the height difference may make the larger nanosphere shadow the gap junction. We intend to obtain more detailed information on morphology with the aid of a helium ion microscopy. This instrument, as used in our earlier work, is highly suited for the task not only because of an excellent spatial resolution but because it does not require the substrates to be coated with an additional layer of an electrically conducting material.

\section{Conclusions}

In this paper, we show that random MFON prepared from randomly adsorbed $\mathrm{SiO}_{2}$ nanospheres are quite effective as SERS substrates. A reduction in the number of Ag nanoparticles per unit area was more than compensated by a morphology more suited for creation of hot spots. The nanosphere diameter and metal deposition thickness are two important parameters that regulate the extinction spectrum and the enhancement ratio. Beside these two parameters, use of random MFON allowed us to explore dependence on the adsorption density and importance of interactions among nanospheres of different diameters. More specifically we observed that the following are important for optimizing SERS performance: (1) introduction of slight space among nanospheres, (2) a silver layer thick enough to bridge the gap between two adjacent layers, and (3) mixing of nanospheres with different diameters.

Another advantage of random MFON is the intrinsically low cost of preparation. It requires neither highly monodisperse nanospheres nor precisely flat surfaces for formation of a nanosphere monolayer, allowing use of inexpensive glass or polymer surfaces as the substrate. It is our hope that random MFON will play an important role in spreading the use of SERS to industrial settings.

\section{Supporting Information}

This material is available free of charge on the Web at http:// www.jsac.or.jp/analsci/.

\section{References}

1. M. Fleischmann, P. J. Hendra, and A. J. McQuillan, Chem. Phys. Lett., 1974, 26, 163.

2. D. L. Jeanmaire and R. P. Van Duyne, J. Electroanal. Chem., 1977, 84, 1.

3. M. Osawa, Top. Appl. Phys., 2001, 81, 163.

4. J. Zhang and J. R. Lakowicz, Optics Express, 2007, 15, 2598.

5. T. Okamoto, F. H'Dhili, and S. Kawata, Appl. Phys. Lett., 2004, 85, 3968.

6. S. Nie and S. R. Emory, Science, 1997, 275, 1102.

7. B. Sharma, R. R. Frontiera, A.-I. Henry, E. Ringe, and R. P. Van Duyne, Materialstoday, 2012, 15, 16.

8. K. Hering, D. Cialla, K. Ackermann, T. Dörfer, R. Möller, H. Schneidewind, R. Matthies, W. Fritzsche, P. Rösch, and J. Popp, Anal. Bioanal. Chem., 2008, 390, 113.

9. S. Lai, N. K. Grady, J. Kundu, C. S. Levin, J. B. Lassiter, and N. J. Halas, Chem. Soc. Rev., 2008, 37, 898. 
10. R. A. Tripp, R. A. Dluhy, and Y. Zhao, Nanotoday, 2008, 3, 31.

11. A. Kudelski, Surf. Sci., 2009, 603, 1328.

12. K. L. Wustholz, A.-I. Henry, J. M. McMahon, R. G. Freeman, N. Valley, M. E. Piotti, M. J. Natan, G. C. Schatz, and R. P. Van Duyne, J. Am. Chem. Soc., 2010, 132, 10903.

13. J. P. Camden, J. A. Dieringer, Y. Wang, D. J. Masiello, L. D. Marks, G. C. Schatz, and R. P. Van Duyne, J. Am. Chem. Soc., 2008, 130, 12616.

14. J. Theiss, P. Pavaskar, P. M. Echternach, R. E. Muller, and S. B. Cronin, Nano Lett., 2010, 10, 2749.

15. K. Yoshida, T. Itoh, H. Tamaru, V. Biju, M. Ishikawa, and Y. Ozaki, Phys. Rev. B, 2010, 81, 115406.

16. J. C. Hulteen and R. P. Van Duyne, J. Vac. Sci. Technol. A, 1995, 13, 1553.

17. C. L. Haynes, A. D. McFarland, M. T. Smith, J. C. Hulteen, and R. P. Van Duyne, J. Phys. Chem. B, 2002, 106, 1898.

18. L. A. Dick, A. D. McFarland, C. L. Haynes, and R. P. Van Duyne, J. Phys. Chem. B, 2002, 106, 853.

19. X. Zhang, M. A. Young, O. Lyandres, and R. P. Van Duyne, J. Am. Chem. Soc., 2005, 127, 4484.

20. C. Farcau and S. Astilean, J. Phys. Chem. C, 2010, 114, 11717.

21. M. Baia, L. Baia, and S. Astilean, Chem. Phys. Lett., 2005, $404,3$.

22. Y.-J. Oh, S.-G. Park, M.-H. Kang, J.-H. Choi, Y. Nam, and K.-H. Jeoung, Small, 2010, 7, 184.

23. H. Takei, J. Vac. Sci. Technol. B, 1999, 17, 1906.

24. H. Takei and M. Himmelhaus, in "Progress in NanoElectro-Optics III", ed. M. Ohtsu, 2005, Springer, Berlin, 57.

25. H. Takei, M. Himmelhaus, and T. Okamoto, Opt. Lett., 2002, $27,1$.
26. M. Himmelhaus and H. Takei, Phys. Chem. Chem. Phys., 2002, 4, 495.

27. H. Takei, N. Bessho, A. Ishii, T. Okamoto, A. Beyer, H. Vieker, and A. Gölzhäuser, Langmuir, 2014, 30, 2297.

28. T. Yamaguchi, T. Kaya, and H. Takei, Anal. Biochem., 2007, 364, 171.

29. T. Yamaguchi, T. Kaya, M. Aoyama, and H. Takei, Analyst, 2009, 134, 776.

30. H. Takei and T. Yamaguchi, Phys. Chem. Chem. Phys., 2010, 12, 4505.

31. H. Takei, J. Saito, K. Kato, H. Vieker, A. Bayer, and A. Gölzhäuser, J. Nanomaterials, 2015, doi:10.1155/2015/ 316189.

32. L. Baia, M. Baia, J. Popp, and S. Astilean, J. Phys. Chem. B, 2006, 110, 23982.

33. H. Wang, C. S. Levin, and N. J. Halas, J. Am. Chem. Soc., 2005, 127, 14992.

34. C. Haginoya, M. Ishibashi, and K. Koike, Appl. Phys. Lett., 1997, 71, 2934.

35. J. Wang, L. Yang, S. Boriskina, B. Yan, and B. M. Reinhard, Anal. Chem., 2011, 83, 2243.

36. A. Gopinath, S. V. Boriskina, W. R. Remasiri, L. Ziegler, B. M. Reinhard, and L. Dal Negro, Nano Lett., 2009, 9, 3922.

37. F. Le, D. W. Brandl, Y. A. Urzhumov, H. Wang, J. Kundu, N. J. Halas, J. Aizpurua, and P. Nordlander, ACS Nano, 2008, 2, 707.

38. Y. Fang, N.-H. Seong, and D. D. Dlott, Science, 2008, 321, 388.

39. M. I. Stockman, L. N. Pandey, and T. F. George, Phys. Rev. $B$, 1996, 53, 2183.

40. J. Kneipp, X. Li, M. Sherwood, U. Panne, H. Kneipp, M. I. Stockman, and K. Kneipp, Anal. Chem., 2008, 80, 4247. 